Please send trade news information and illustrations to Arveen Bajaj at the $B D J$, Nature Publishing Group, The Macmillan Building, 4-6 Crinan Street, London N1 9XW. Trade news is provided as a service to readers using text and images from the manufacturer, supplier or distributor and does not imply endorsement by the $B D J$. Normal and prudent research should be exercised before purchase or use of any product mentioned.

\section{Innovation in luxation}

Sullman Surgical Limited has introduced a new style of universal luxating instrument, the Sullman- Luxatome. The patented angled design of the Sullman- Luxatome allows the clinician to apply directed luxating forces buccally, lingually and palatally, with a greatly reduced risk of slippage in use. The distinctive design allows effective application of luxating forces to teeth and roots that are compromised mesially and distally by caries or trauma, are tilted, rotated, impacted, crowded, as well as in cases of limited access. According to the company, the instrument is particularly useful in the luxation of carious or fractured roots that have lost most or all of their coronal structure. In addition, the design allows timesaving, retention of good access, vision and illumination, whilst also reducing stress on the clinician's wrist and on the patient's musculoskeletal system.

Reader response number 51

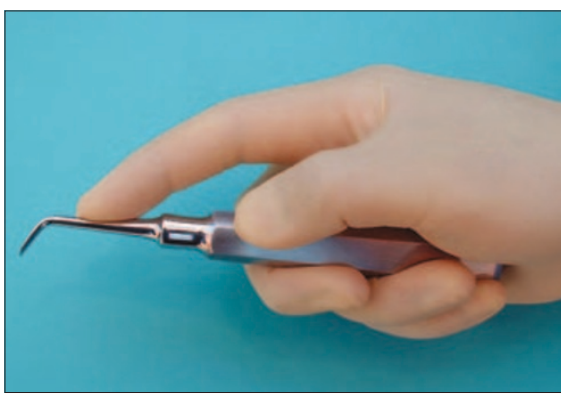

\section{Seeing clearly}

DP Medical has introduced the new Oakley "M" frame to compliment SurgiTel loupes. Full adjustability of height, angle of view or declination is vital in achieving the correct working posture while balance, comfort and weight are also of great importance in binocular loupes worn for extended periods.

The Oakley "M" frame has been designed for balance and lightness. These new fashionable frames also provide excellent protection with wrap round lenses which can incorporate a prescription if required. Fibre optic and LED lights are also

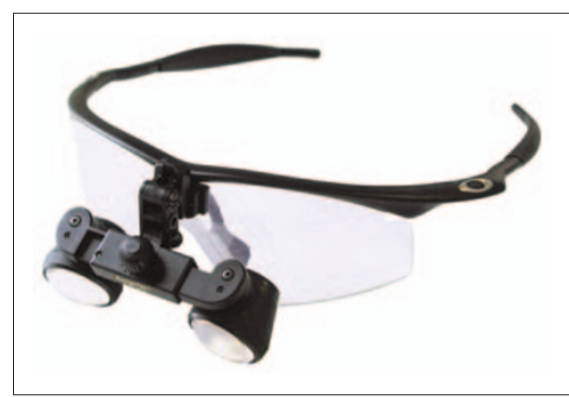

available to compliment your loupes for optimum illumination.

Reader response number 50

\title{
Splash of colour in waiting rooms
}

A mouth watering range of new waiting room materials have been produced to stimulate interest in Sonicare which feature the theme 'A healthier lifesmile'. The campaign highlights the importance of a healthy lifestyle - which naturally includes caring for the teeth. To complement the campaign, a £10 cash-back incentive has been initiated to stimulate extra interest. The materials for the dental practice include posters, A4 and A5 sized showcards, leaflets which can be posted or inserted in a leaflet dispenser and patient appointment cards.

Also free for every practice are three easy-to-read-and-understand leaflets which have been produced to educate patients about the risks of periodontal

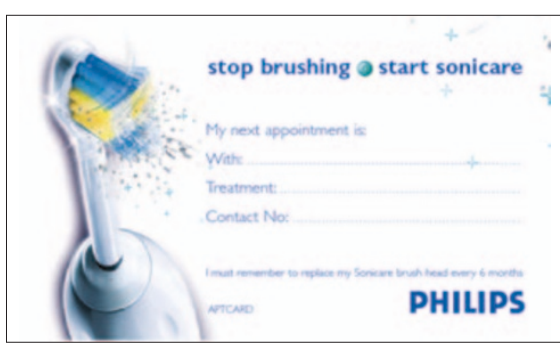

disease and coronary heart disease, diabetes and preterm low birth weight babies. These are complemented by a general overarching leaflet about the newest Sonicare, the e9000 - which explains the benefits of the new mini brush head and two speed power handle.

Reader response number 52

\section{Keeping patients informed}

Canadian based company, Patient News Publishing, has recently launched its Patient and Neighbourhood Newsletter Programmes to the UK dental marketplace.

The newsletter programmes, which follow a unique tried and tested system, have been designed to educate, inform and expand patient knowledge. It believes this provides increased levels of understanding between dentist and patient, which in turn prompts further questions about cosmetic and preventive dentistry services.

Existing patients receive information in the comfort and security of their homes to build and strengthen patient loyalty and prospective patients who live around the practice are targeted with the neighbourhood newsletter programme.

Reader response number 53

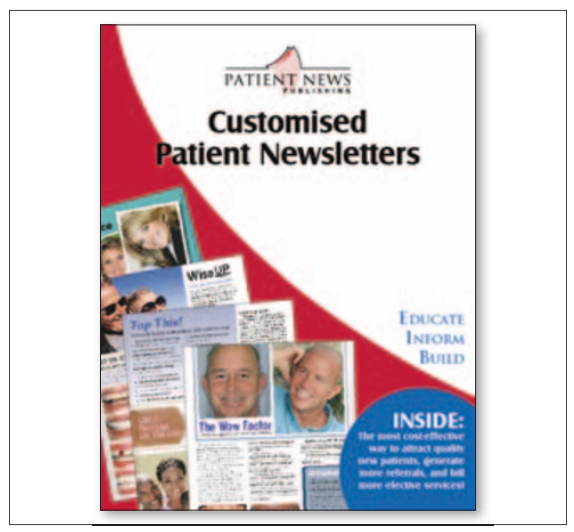



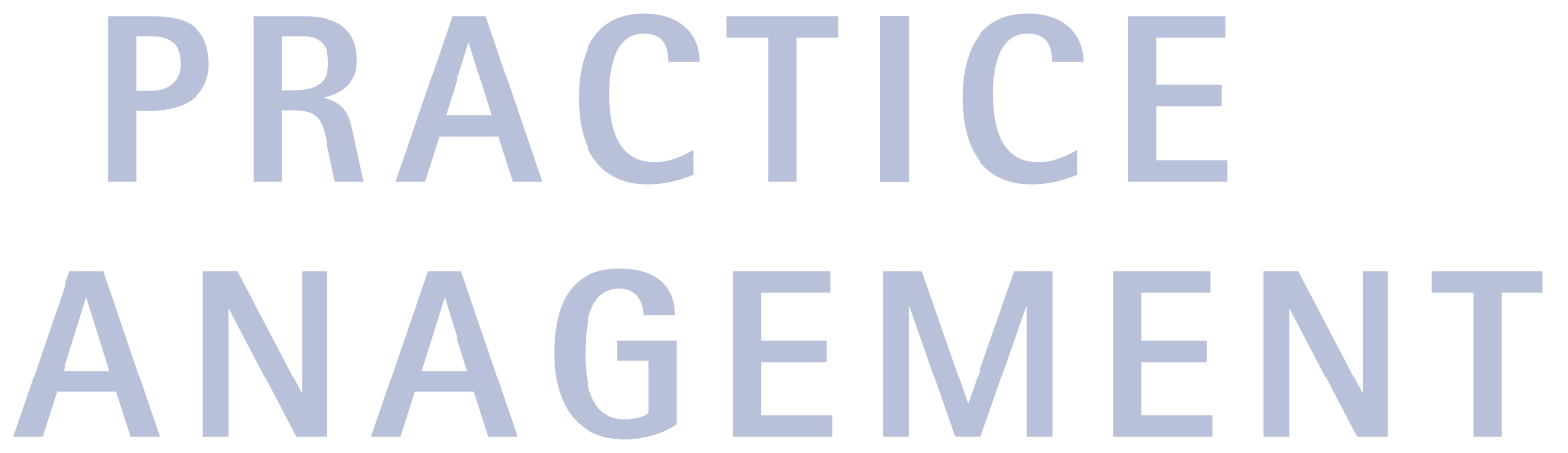

\section{Practical workwear solutions}

Hejco's Oxford Collection is presented in a range of attractive colour combinations to offer a soft, pleasantly relaxed impression. This range of blouses and unisex shirts is designed to complement all Hejco trousers, allowing you to choose the most suitable combination for your needs. The Oxford range's comfortable, loose fitting design permits unrestricted movement but also ensures that nothing is revealed when you bend or stretch!

The Twill Range of work wear is fashioned for the hustle and bustle of life in busy modern practice. It's loose fitting, robust styling is designed to cope with the unexpected as well as the day to day routine of practice life. Although manufactured in a stronger fabric, it still hangs well from the body and gives a flattering fit. Matching trousers are also available for a coordinated look. The range is a practical workwear solution, furnished with a number of pockets which includes a specially designed, divided breast pocket ideal for keeping pens organised.

Reader response number 55

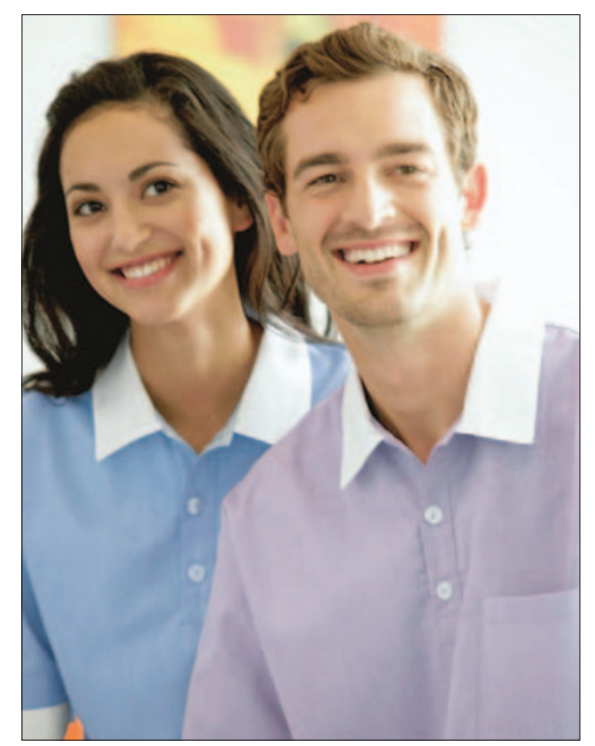

Enjoying the view

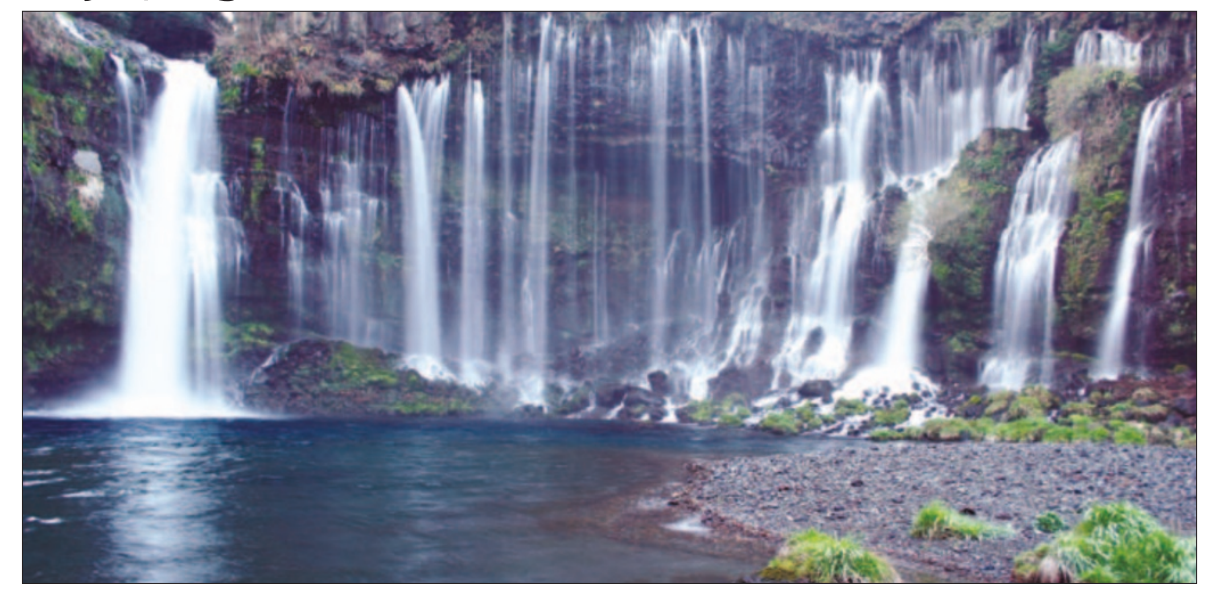

Designer Dental has launched an additional range to their poster collection with a choice of ceiling posters. These posters are in full colour and come in three sizes with six different designs, are bright, vibrant and cover a range of scenic and world features. They have also been designed to suit most practice colour schemes so they can easily blend into surgery surroundings. The posters can be viewed and ordered via their web site www.designerdental.co.uk. Reader response number 54

\section{Full range of surgery design}

Henry Schein's equipment brochure is able to accommodate all surgery design and budgetary requirements and includes three ranges symbolised by the Silver, Gold and Platinum ranges. These provide everything from the wide range of entry level equipment and products in the Silver Range to some of the best surgery equipment in the Platinum Range. It also offers full support both pre and post purchase including staff training to ensure the correct use of your new equipment.

Reader response number 56

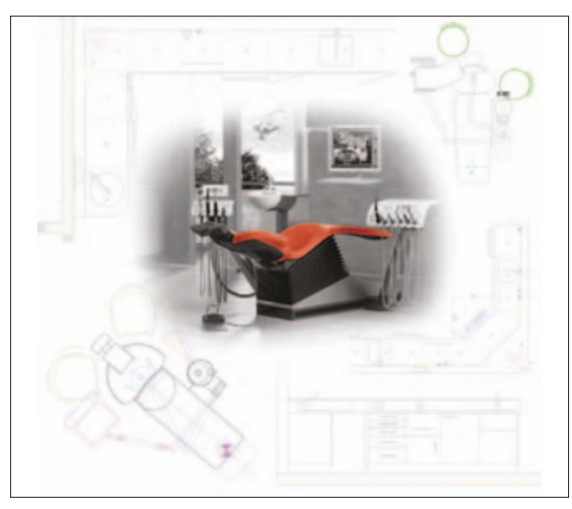

\section{Computerised protection}

The role and responsibilies of those in the dental and healthcare professions with regard to child abuse has recently been documented in the wake of the Victoria Climbié tragedy.
Software of Excellence has worked with the relevant authorities and can offer a customised screen to record and track such information.

Reader response number 57 


\section{Treatment consent protocols}

If a patient does not understand the treatments you are suggesting or why they need them, they will be unable to give informed consent. To avoid potential litigation as a result of this, Admor, in association with Dental Protection, has developed a series of Treatment Consent protocols to ensure that patients are fully informed about their treatment plans. In particular, the NCR (no carbon required) pad designed for crowns and bridges provides a variety of advice and information to ensure the dentist gains consent in an appropriate manner and that the patient has access to information needed to understand exactly what they are consenting to.

A set of five sheets provides information and guidance on commonly asked questions such as what crowns and bridges are and who needs them. There is also a two set consultation form (a copy each for patient

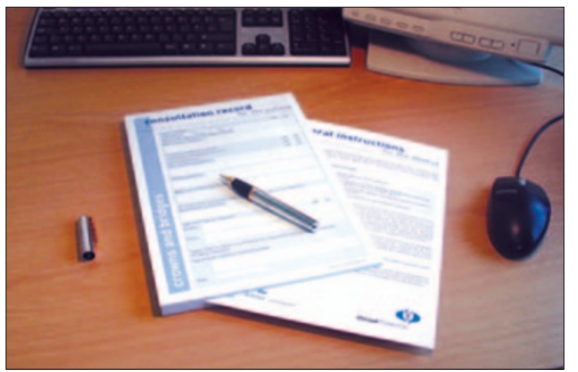

and dentist) on which details of the consultation can be recorded, with space for a patient signature.

The dentist can file their copy with patient records or scan it onto a computer-based record, and the patient copy has simple, easy to follow advice on the reverse to give further detailed information to the patient and pre and post treatment advice.

Reader response number 58

\section{Presentation of treatment}

Kodak's Patient Journey enables you to create a clear presentation of treatment to aid patient understanding. All you need to do is enter the treatment within R4 in the normal way, click into 'Patient Journey' and instantly the presentation of treatment is transformed into a clear flowchart. Whether it is a short, simple course of treatment or a complex case with treatments that are not necessarily made until some way through the course, many patients get very confused as to when and where treatments will occur. The Patient Journey offers the solution as a new way to quickly and simply keep the patient in the picture by producing an illustrated, fully customisable flowchart that the patient can take away with them.

Reader response number 59

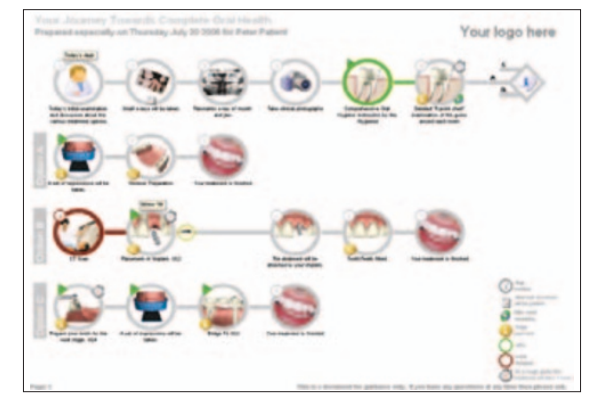

\section{No more missed appointments}

The Appointment Prompting System (APS) from Ecce Media, the web and ecommerce development company can

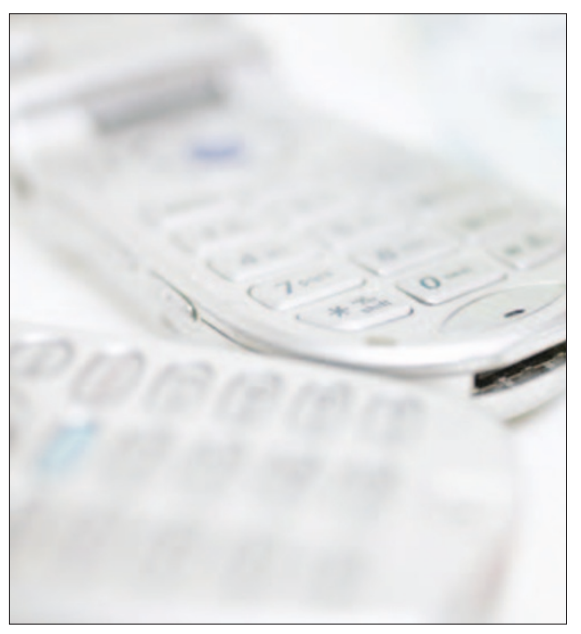

reduce costs in a practice by automating manual processes, allowing staff to concentrate on more productive tasks, the company claims.

The APS is an easy to use online appointment booking system with an automated reminder service using SMS text or email. It is an internet based solution requiring a broadband connection, such as BT Broadband and a standard computer, which can dramatically reduce missed appointments through its preappointment reminders.

The system also provides statistical analysis on appointments and reports on productivity by practitioner. This helps quantify costs of missed appointments and may assist in making informed decisions for the business.

Reader response number 60 